


\title{
Diseño de un Modelo de Inventarios (EOQ) y Simulación en Redes de Petri para una empresa artesanal productora y comercializadora de trapeadores
}

\author{
María José Torres Mancera ${ }^{1}$, Julio Adrián Orozco Guevara², \\ Angélica Torregroza Espinosa ${ }^{3}$, Desmond Mejía Ayala ${ }^{4}$
}

\section{Resumen}

El estudio presentado en este artículo diseña una propuesta de mejora para el sistema de abastecimiento e inventarios de una empresa artesanal productora y comercializadora de trapeadores en Sincelejo, Sucre. Esta propuesta tiene el objetivo de minimizar los costos en el inventario de materias primas y generar mayores ingresos al negocio, en cuanto a utilidades monetarias. Todo esto mediante estrategias de planificación del control de abastecimiento o compra de materiales. Para ello, se clasifican los productos mediante el método ABC, de acuerdo al aporte de los mismos en las ventas totales de la empresa, se diseña un modelo de cantidad óptima de pedido (EOQ) para administrar la información de las materias primas e insumos que se utilizan frecuentemente en el sistema productivo y que se deben tener a la mano en las bodegas de almacenamiento. Lo anterior permite establecer los registros y pronósticos de los productos, así como determinar los lotes óptimos a pedir, los puntos de reorden de

1 Ingeniera Industrial. Corporación Universitaria del Caribe-CECAR. Correo: maria.torresm@cecar.edu.co; Orcid: https://orcid.org/0000-0003-1872-8553

2 Ingeniero Industrial. Corporación Universitaria del Caribe-CECAR. Correo: julio.orozco@cecar.edu.co; Orcid: https://orcid.org/0000-0002-5698-1261

$3 \mathrm{PhD}(\mathrm{c})$ en Proyectos; Magíster en Ciencias Agroalimentarias; Ingeniera Agroindustrial; Corporación Universitaria del Caribe-CECAR. Líder del grupo gestión de la innovación empresarial y social-GIES. Investigadora Asociado Minciencias. Correo: angelica.torregroza@cecar.edu.co; Orcid: https://orcid.org/0000-0002-8948-0914 $4 \quad$ Candidato a Doctor en Educación por UCV Perú. Magíster en Gestión de operaciones UPC España. Magister en Dirección de operaciones y logística UPC Perú. Ing. Industrial UNMSM Perú. Docente de pre grado y postgrado en UNMSM - USIL UPC. Correo: dmejiaa@ucv.edu.pe; Orcid: https://orcid.org/0000-0003-3749-3569 
pedidos en un determinado tiempo, asegurando los menores costos y el momento justo en que se debe generar la orden de pedidos a los proveedores. De manera experimental, el modelo describe el sistema productivo de trapeadores artesanales en la herramienta HP Sim (Redes de Petri), donde se simula el proceso productivo, obteniendo como resultado que el inventario arrojado por el EOQ garantiza el funcionamiento del sistema, abarcando la producción y generando los inventarios requeridos para tener el flujo de fabricación constante.

Palabras clave: bastón, máquina artesanal, pabilo de algodón, sistema productivo, trapeador.

\section{Design of an Inventory Model of Optimal Quantity of Orders (EOQ) and Simulation in Petri Nets of a handicraft company producer and marketer of mops}

\section{Abstract}

The study carried out in the article designs an improvement proposal for the supply system and inventories of an artisan production and marketing company of mops in the city of Sincelejo Sucre, with the objective of minimizing costs in the inventory of raw materials and generating greater income in terms of monetary profits to the business, all this through planning strategies of supply control or purchase of materials. To do this, the products are classified using the $A B C$ method, according to the contribution of the same in the total sales of the company. Next, a model of optimal quantity of order (EOQ) is designed to manage the information of the raw materials and supplies that are frequently used in the production system and that should be kept at hand in the storage warehouses, establishing the records and forecasts of the products and determine the optimal batches to be ordered, the reorder points of orders in a bucket time, ensuring the lowest costs and the right moment in which must generate the order of orders to suppliers. Experimentally, the model describes the productive system of artisanal mops, in the HP Sim tool (Petri nets), where the production process is simulated, obtaining as a result that the inventory thrown by the EOQ guarantees the operation of the system covering the production and generating the inventories required to have the constant manufacturing flow.

Keywords: walking stick, Artisan machine, cotton wick, Production system, Mop. 


\section{Introducción}

El interés de la humanidad por la limpieza y el orden constante va en aumento. De allí que tanto en los hogares como en todos los entornos en que se desenvuelven las personas sea inevitable mantener cada cosa limpia. Es por ello que, cada vez más, se acrecienta la producción artesanal de herramientas de aseo como traperos, escobas, cepillos, entre otros, los cuales permiten tener, a un bajo costo, diferentes utensilios para mantener el espacio en el que se convive en condiciones óptimas de higiene.

Al cierre de 2012, la producción de implementos de aseo representó el $1.5 \%$ del PIB nacional y el $12.4 \%$ del PIB industrial de Colombia, ocupando el quinto lugar del mercado de cosméticos y artículos de aseo en Latinoamérica, con un consumo per cápita de US\$79,8, se estima que en 2020 la producción de esta industria representará US\$5.754 millones.

Actualmente, Bogotá es el principal centro de operaciones de las compañías del sector, pues cerca del $40 \%$ de la industria se encuentra concentrada allí; 30\% en Antioquia; y otro 30\%, en el Valle del Cauca (Dinero, 2017). Asimismo, el crecimiento de las ventas del sector de productos de línea del hogar y aseo se aceleró durante 2012. Después de este cambio en el crecimiento de las ventas del sector, el ritmo de producción también se aceleró, razón por la cual cada día se están fabricando más bienes de este tipo (Benchmark, 2016).

"De la misma manera como los productos han evolucionado, la logística en el mundo competitivo está cambiando a unas velocidades sorprendentes" (Acero, 2006). Los empresarios colombianos tienen que pensar con una mentalidad más amplia, lo cual implica que cada uno de ellos debe acoger estrategias logísticas como la administración de su cadena de suministro, involucrando los distintos tipos de eslabones para que trabajen en forma conjunta, donde el flujo y abastecimiento de materiales se encuentre relacionado a la velocidad del mercado.

La creación de microempresas de productos de aseo es la que abastece la demanda en el departamento de Sucre, puesto que se busca la manera de fabricar utensilios accesibles para la ciudadanía, al tiempo que se genera trabajo para personas que no cuentan con una oportunidad en el mercado laboral. 
En la empresa objeto de estudio existen fallas en el registro y control de la materia prima y de la mercancía o artículos terminados, ya que no se lleva el control de inventarios y stock; por esta razón, la empresa requiere de un sistema que controle realmente el inventario que se necesita para fabricar los productos demandados. Con el diseño de este sistema se evitarían los problemas que actualmente se presentan a menudo en la empresa, como el no saber con qué inventario se cuenta, el agotamiento de alguna materia prima que se cree tener en stock, demasiadas materias primas de la misma referencia, entre otros. Lo anterior ocasiona caos en el área de producción de la empresa. (Laveriano, 2010)

Por todo lo anterior, elaborar un diseño de control de inventario EOQ (Economic Order Quantity), cantidad óptima de pedido, permite la interacción directa entre el área de ventas, de producción y los inventarios, con el objetivo de determinar la cantidad óptima de pedido y el instante en que debe pedirse (Holguín, 2005). Esto ayuda a coordinar eficientemente la cadena de suministro con el proveedor, pasando por la mejora de los procesos internos de la empresa como lo describe Laveriano (Laveriano, 2010) en su informe titulado Importancia del control de inventarios. Con tal propuesta se busca satisfacer la necesidad de toda la población Sucreña, mediante productos de buena calidad, en el tiempo requerido, en las cantidades necesarias y a buen precio, para ser utilizados tanto en los hogares como en cualquier otro recinto.

\section{Materiales y métodos}

Producto de la visión y emprendimiento del señor Carlos Lugo, en Julio del 2009, se origina la idea de crear una empresa que produzca trapeadores de manera artesanal, con la intención de aprovechar una oportunidad de negocio que presenta el mercado de Sincelejo y Sucre. La microempresa es de origen familiar, se encuentra ubicada en el barrio El Bosque de la ciudad de Sincelejo, Sucre. Actualmente, su proceso productivo se basa en la fabricación de traperos mediante máquinas artesanales. Su mano de obra son personas empíricas, pero experimentadas en cada una de las actividades que se desarrollan en la línea de producción. 


\section{Productos}

Trapeadores de pabilo de algodón blanco.

Figura 1

Diagrama BOM.

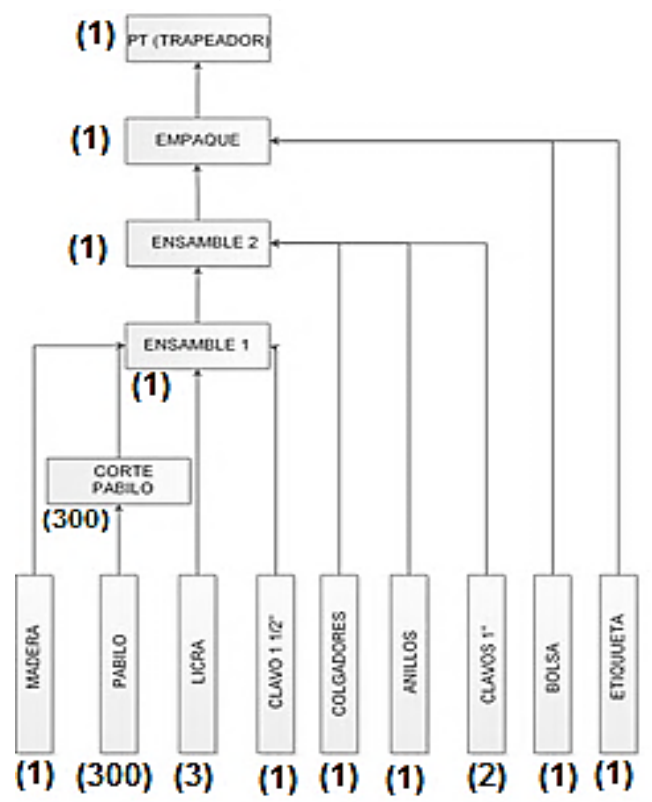

Nota. Fuente: construcción propia.

\section{Modelamiento y simulación}

La estructura del modelo es muy importante en este trabajo, pues por medio de esta se pueden incluir las variables que se desean abarcar en el estudio, tales como las unidades que se pueden producir en un horizonte de tiempo, la materia prima, insumos requeridos para fabricar cierta cantidad de productos y demás aspectos involucrados en cada uno de los centros de trabajo (Gaviria \& Zapata, 2006). Para escoger el producto se desarrolló un análisis de $\mathrm{ABC}$ basado en la información suministrada por los trabajadores y el propietario del negocio. La gráfica 4 describe que el trapero de mayor venta es el antes mencionado. 
Figura 2

Gráfica aplicación $A B C$.

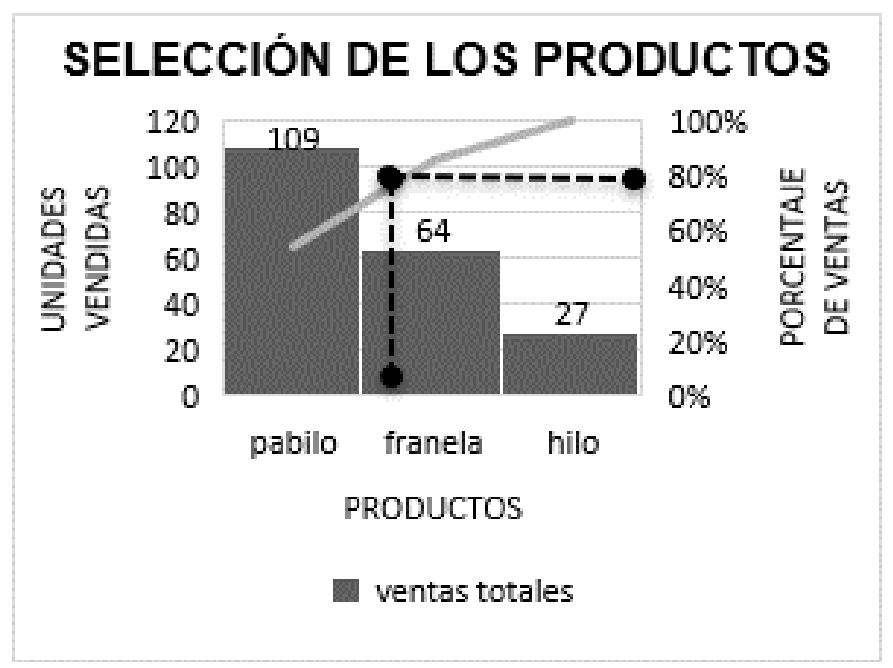

Nota. Fuente: construcción propia.

\section{Planeación cantidad óptima de pedido (EOQ)}

Para el diseño de un sistema MRP es necesario conocer la demanda de los productos que ofrece la empresa, con ella se puede calcular de forma oportuna y precisa la demanda dependiente de las cantidades de materias primas e insumos, generada por las demandas de los productos finales. En contraste, un EOQ facilita el control de abastecimiento de materia prima en cuanto a cantidades, tiempos de pedido, puntos de reorden de materias primas, costos de pedido, costos de mantener, entre otros aspectos (Holguín, 2005).

El trabajo de esta investigación está basado en el modelo EOQ, por medio del cual se establecieron y denominaron las variables que influyeron en el estudio, como son:

- Las cantidades demandadas del producto (D).

- El Lead Time o tiempo de entrega por parte de los proveedores.

- El inventario, el cual es reordenado cuando llega a cero.

- En este estudio no se consideran descuentos por volumen de pedido. 
- Los costos de ordenar un pedido, el cual es igual al costo de realizar un pedido (S), multiplicado por la demanda del producto (D), entre la cantidad de unidades de pedido (Q), representados en la ecuación 1.

$$
\text { Costo ordenar }=S * \frac{D}{Q}
$$

Los costos por comprar suministros, los cuales están dados por el costo unitario de compra (C), multiplicado por la demanda del producto (D). Ver ecuación 2.

\section{Costo de Compra $=C^{*} D$}

Los costos unitarios de mantener el inventario (H), que está dado por el costo de mantener inventario expresado en porcentaje (i), multiplicado por el costo unitario de compra (C). Ver ecuación 3.

\section{Costo de Mantener Inventario $=i^{*} \mathrm{C}$}

Tamaño óptimo de pedido (Q), el cual se expresa mediante la ecuación 4 del modelo de cantidad económica (EOQ) de pedido.

$$
Q^{*}=\sqrt{\frac{2 S D}{H}}
$$

Seguidamente, se realiza la proyección de la demanda para los siguientes años con base a las ventas registradas en años anteriores, utilizando el método de análisis de regresión lineal según plantean en la referencia (Richard B. Chase, 2009), debido a que estos se asemejan a un comportamiento lineal en sus ventas. La tabla 1 muestra los resultados del pronóstico.

Tabla 1

Pronóstico de la demanda.

\begin{tabular}{ccccc}
\hline \multicolumn{5}{c}{ Demanda pronosticada años siguientes } \\
AÑO & $\begin{array}{c}\text { Docenas } \\
\text { Año }\end{array}$ & $\begin{array}{c}\text { Docenas } \\
\text { Mes }\end{array}$ & $\begin{array}{c}\text { Docenas } \\
\text { Semana }\end{array}$ & $\begin{array}{c}\text { Docenas } \\
\text { Día }\end{array}$ \\
\hline 2018 & 3501 & 438 & 109 & 16 \\
2019 & 3853 & 482 & 120 & 17 \\
2020 & 4206 & 526 & 131 & 19 \\
2021 & 4558 & 570 & 142 & 20 \\
2022 & 4910 & 614 & 153 & 22
\end{tabular}




\begin{tabular}{ccccc}
\hline \multicolumn{5}{c}{ Demanda pronosticada años siguientes } \\
AÑO & $\begin{array}{c}\text { Docenas } \\
\text { Año }\end{array}$ & $\begin{array}{c}\text { Docenas } \\
\text { Mes }\end{array}$ & $\begin{array}{c}\text { Docenas } \\
\text { Semana }\end{array}$ & $\begin{array}{c}\text { Docenas } \\
\text { Día }\end{array}$ \\
\hline 2023 & 5262 & 658 & 164 & 23 \\
\hline
\end{tabular}

Nota. Fuente: construcción propia.

\section{Construcción del modelo}

El modelo fue construido en Excel y HPSim redes de Petri, en el cual se usa un modelo de producción bajo pedido EPP, donde se seleccionan los recursos necesarios para fabricar el promedio semanal del trapero según las condiciones estudiadas en la empresa.

Las variables de decisión establecidas en el modelo son:

\section{Tabla 2}

Variables de decisión.

\begin{tabular}{ll}
\hline Variables de decisión \\
\hline 1. & Unidades producidas bajo pedido \\
2. Cantidad óptima de pedido de materia prima \\
3. Punto de reorden del pedido \\
4. Costos totales de pedidos y mantener \\
\hline
\end{tabular}

Nota. Fuente: construcción propia.

Luego se simula en HP Sim bajo el entorno con una línea de producción, donde las redes de Petri permiten crear escenarios que ayudan a observar el comportamiento de estos en sus distintas fases y lograr varias alternativas de solución. Esta simulación de escenario utiliza nodos o lugares representados mediante plazas (circunferencias), así como transiciones representadas por rectángulos, los antes mencionados se unen mediante arcos o flechas. Todo lo anterior resulta necesario para abarcar los procesos, los cuales se representan mediante triángulo compuesto por plazas, arcos y transiciones, y agregando un punto en el interior del círculo. La figura 7 describe los elementos principales para simular en HPsim. 
Figura 3

Elementos de redes de Petri.

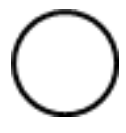

Plaza

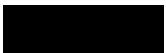

Transición
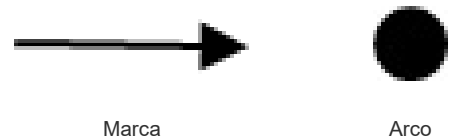

Arco

Nota. Fuente: construcción propia.

\section{Resultados y discusión}

La aplicación del modelo EOQ en la siguiente tabla (3) describe el lote económico de pedido, es decir, las cantidades a pedir por parte de la empresa al proveedor que le traerá menores costos, cuyo lote está en 609 kilogramos de pabilo de algodón; el punto de reorden de la materia prima es de 173 kilogramos; los días que debe haber entre cada pedido, los cuales están en alrededor de 15 días entre cada una; entre otros aspectos.

Del mismo modo, la siguiente gráfica describe la comparación de los costos entre las cantidades óptimas de pedido arrojadas por el modelo EOQ y diferentes cantidades de lotes de pedido, los cuales representan los costos totales anuales por pedir y mantener el inventario. Se observa que el lote a pedir dado por el modelo EOQ es el que representa los menores costos en comparación con los demás, los cuales se encuentran en \$289.828; el costo por pedir se encuentra en $\$ 144,882$ al año; y el de mantener inventario, en $\$ 144,945$ al año.

Tabla 3

Modelo EOQ pabilo de algodón.

\section{Modelo cantidad económica de pedido (EOQ) pabilo de algodón} Ítem

Datos Detalle

Demanda (D)

Demanda (D)

Costo de ordenar (S)

Costo de mantener $(\mathrm{H})$

$\mathrm{N}^{\circ}$ de días de trabajo

Costo (C)

Cantidad óptima de pedido $\mathrm{Q}^{*}$

3501

12605

$\$ 7,000$

$\$ 476$

298

$\$ 1,519$

608.9
Docenas Anuales

Kg anuales de pabilo

Por orden

Anual (kg)

Por año

Por Gramos/ud.

Se deben pedir $609 \mathrm{~kg}$ por orden 


\section{Modelo cantidad económica de pedido (EOQ) pabilo de algodón}

Ítem

Datos

Detalle

Número esperado de órdenes $(\mathrm{N})$

Tiempo esperado entre órdenes

(L)

Punto de reorden (R)

Tiempo de entrega proveedor

Tiempo de entrega proveedor

Costo total (CT)

$\$ 19,331,770$

Costo de ordenar

Costo de mantener
172.7 Cuando el nivel de inventario esté en $173 \mathrm{~kg}$, se debe colocar una nueva orden

5.0 Tiempo de entrega del proveedor en días

0.014 Tiempo de entrega del proveedor en años

El costo total anual de inventario es de $\$ 19331771$

Se realizan 21 órdenes de pedido

El tiempo entre órdenes es de 15 días

El costo anual de ordenar es de $\$ 144,882$

$\$ 144,945$ El costo anual de mantener es de $\$ 144914$ al año

Nota. Fuente: construcción propia.

Figura 4

Gráfica comparación costos totales.

\begin{tabular}{|c|c|c|}
\hline \multicolumn{3}{|c|}{$\begin{array}{l}\text { Comparación de costos modelo EOQ y } \\
\text { lotes arbitrarios de pabilo de algodón }\end{array}$} \\
\hline \begin{tabular}{r}
$\$ 450.000$ \\
$\$ 400.000$ \\
$\$ 350.000$ \\
$\$ 300.000$ \\
$\circ \$ 250.000$ \\
\multirow{O}{O}{$\$ 200.000$} \\
$\$ 150.000$ \\
$\$ 100.000$ \\
$\$ 50.000$ \\
$\$ 0$
\end{tabular} & $\$ 289.828$ & $\frac{\text { Costo }}{\text { Ordenar }}$ \\
\hline & $\begin{array}{l}6^{\circ} 6^{\circ} \\
\text { CANT }\end{array}$ & $\underset{2^{\circ}}{2^{\circ}} \sqrt[3]{ }^{\circ} \nabla^{\circ}$ \\
\hline
\end{tabular}


Nota. Fuente: construcción propia.

En la figura 5 se gráfica el punto de reorden del pedido, lo que quiere decir que la orden de pedido de pabilo de algodón debe tener un tiempo estimado de 15 días entre cada pedido y un tiempo de entrega de 5 días por parte del proveedor.

Figura 5

Gráfica punto de reorden.

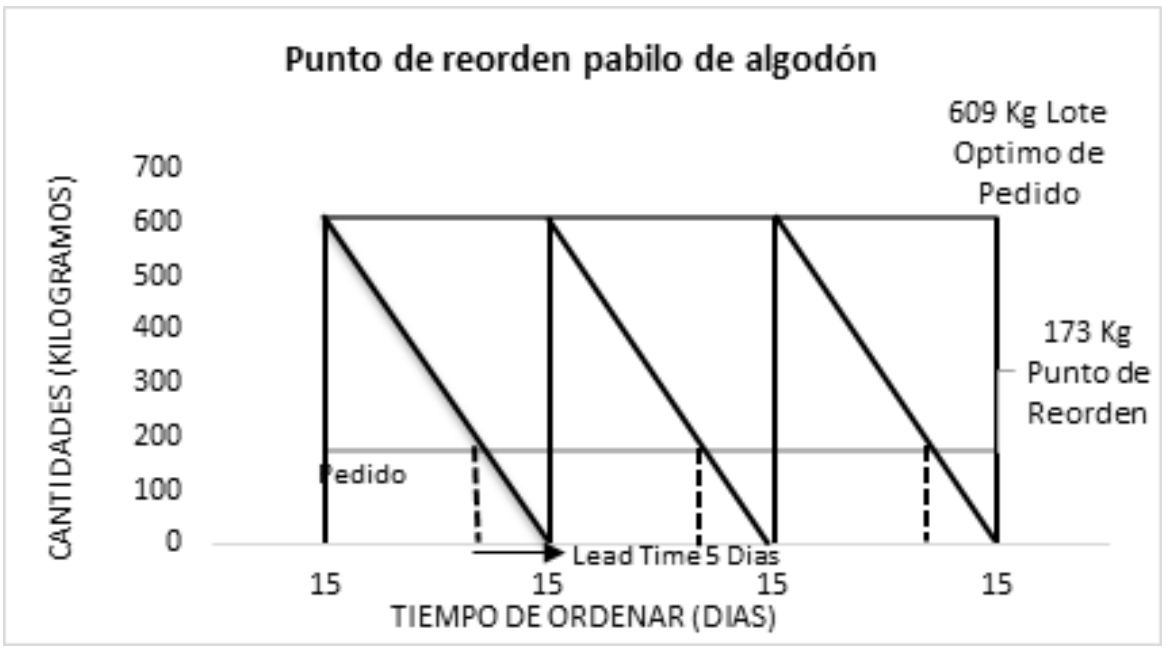

Nota. Fuente: construcción propia.

A cada materia prima proveniente de la ciudad de Medellín, Antioquia, (bastones de madera, colgadores, anillos y licra de amarre), se les realizó lo descrito anteriormente en la tabla 3 (Modelo cantidad económica de pedido EOQ), la figura 4 (Gráfica de comparación costos totales) y la figura 5 (Gráfica punto de reorden).

En los apéndices A, B, C, D y E anexos se describen las comparaciones entre las cantidades del lote económico de pedido (se subraya en negrilla) y las cantidades de otros lotes arbitrarios de pedido.

Al comparar los costos en cada una de las tablas de los apéndices anteriormente mencionados, se observa que el EOQ es el que presenta menores costos totales en comparación con los diferentes tamaños de lote a pedir, lo cual describe que el modelo sí está arrojando los resultados que garantizan que el lote de pedido económico es el que acarrea menores costos. 
En el apéndice $\mathrm{F}$ se describe el resumen de todos los costos de las materias primas necesarias para la elaboración del trapeador, como se evidencia en el diagrama BOM (figura 2).

En el apéndice $\mathrm{G}$ se describe el resumen de todos los puntos de reorden de las materias primas necesarias para la elaboración del trapeador, como se evidencia en el diagrama BOM (figura 2).

Durante la simulación en el software HP Sim se arma la estructura de la línea de producción como se trabaja en el sistema real, poniendo en práctica las unidades de abastecimiento dadas en el modelo EOQ realizado, dando como resultado las mismas cantidades de lote económico e inventarios que se obtienen para la producción de un lote de pedido promedio de 109 docenas a la semana, es decir, produce las 109 unidades consumiendo las materias primas necesarias y dejando el resto en los inventarios.

\section{Figura 6}

Simulación redes Petri Software HP Sim.

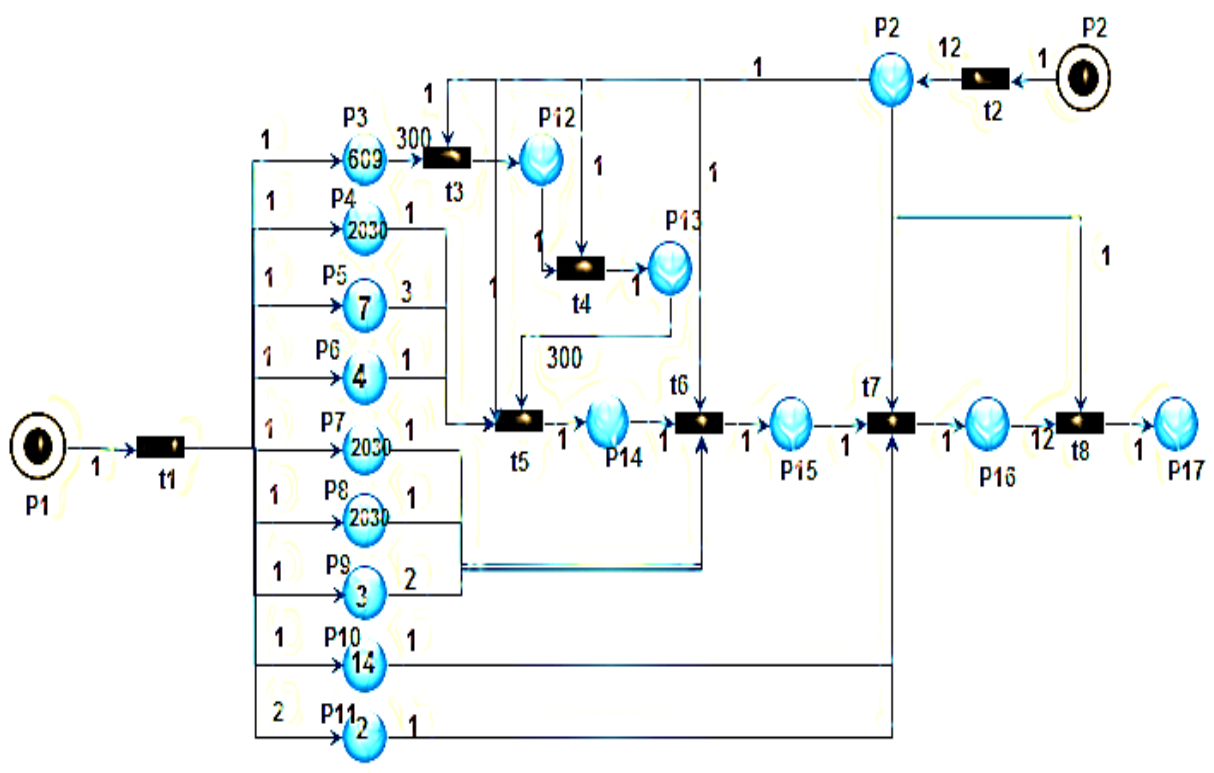

Nota. Fuente: construcción propia.

Las tablas representadas seguidamente describen cada una de las estructuras usadas en la figura 8, su significado y la interpretación de cada elemento empleado en la simulación realizada con redes de Petri, bajo el modelo EPP y EOQ señalado a lo largo del artículo. 
Tabla 4

Representación de los lugares de la Redes Petri.

\begin{tabular}{cl}
\hline Lugares & \multicolumn{1}{c}{ Descripción } \\
\hline P1 & Cantidad a pedir al proveedor \\
P2 & Cantidad demanda/semana \\
P3 & Bodega Pabilo de Algodón EOQ $609 \mathrm{~kg}$ \\
P4 & Bodega Bastones Madera EOQ $2030 \mathrm{uds}$. \\
P5 & Bodega Licra de amarre EOQ $7 \mathrm{~kg}$ \\
P6 & Bodega Clavos 1,5” 4 cajas por 700 uds. \\
P7 & Bodega Colgadores EOQ 2030 uds. \\
P8 & Bodega Anillos EOQ 2030 uds. \\
P9 & Bodega Clavos 3 cajas por 900 uds. \\
P10 & Bodega Bolsas 14 pacas por 100 uds. \\
P11 & Bodega etiquetas 2 pacas por 1000 uds. \\
P12 & Cantidad de pabilo transportado por el operario \\
P13 & Cantidad de pabilo cortado por el operario \\
P14 & Cantidad de trapeadores ensamblados (ensamble 1) \\
P15 & Cantidad de trapeadores ensamblados (ensamble 2) \\
P16 & Cantidad de trapeadores empacados \\
P17 & Cantidad de trapeadores embalados \\
\hline
\end{tabular}

Nota. Fuente: construcción propia.

Tabla 5

Representación de las transiciones de redes de Petri.

\begin{tabular}{cl}
\hline Transiciones & \multicolumn{1}{c}{ Descripción } \\
\hline $\mathbf{t 1}$ & Sistema EPP (Pedido al proveedor) \\
$\mathbf{t 2}$ & Sistema EPP (Pedido del cliente/semana) \\
t3 & Transporte de pabilo a zona de corte \\
$\mathbf{t} 4$ & Corte de pabilo en 300 gramos \\
$\mathbf{t 5}$ & Ensamble trapero (pabilo, madera, licra, \\
& clavos) \\
$\mathbf{t 6}$ & Ensamble colgador, anillos, clavos \\
$\mathbf{t 7}$ & Empacado de traperos/ ud. \\
$\mathbf{t 8}$ & Embalaje por docenas \\
\hline
\end{tabular}

Nota. Fuente: construcción propia. 


\section{Tabla 6}

Representación de los arcos de las redes de Petri.

\begin{tabular}{cl}
\hline Arcos & Descripción \\
\hline P1-t1 & 1 cantidad de pedido a enviar el proveedor \\
t2-P2 & 1 cantidad de demanda semanal 109 docenas \\
t1-P3 & 1 cantidad de pedido a enviar el proveedor $609 \mathrm{~kg}$ \\
t1-P4 & 1 cantidad de pedido a enviar el proveedor $2030 \mathrm{uds}$. \\
t1-P5 & 1 cantidad de pedido a enviar el proveedor 4 cajas \\
t1-P6 & 1 cantidad de pedido a enviar el proveedor $7 \mathrm{~kg}$ \\
t1-P7 & 1 cantidad de pedido a enviar el proveedor $2030 \mathrm{uds}$. \\
t1-P8 & 1 cantidad de pedido a enviar el proveedor $2030 \mathrm{uds}$. \\
t1-P9 & 1 cantidad de pedido a enviar el proveedor 3 cajas \\
t1-P10 & 1 cantidad de pedido a enviar el proveedor 14 pacas \\
t1-P11 & 1 cantidad de pedido a enviar el proveedor 2 pacas \\
P3-t3 & 300 transporte de pabilo por ud. de trapero 300 gr
\end{tabular}

t3-P12 1 cantidad de pabilo almacenado sin cortar

P12-t4 1 cantidad de pabilo cortado 300gr/ud.

t4-p13 1 cantidad de pabilo cortado almacenado

p13-t5 1 cantidad ensamble 1 de traperos

p4-t5 1 cantidad de madera a ensamblar

p5-t5 1 cantidad de licra a ensamblar

p6-t5 1 cantidad de clavos a ensamblar

t5-p14 1 cantidad de traperos ensamblados a almacenar

p7-t6 1 cantidad de colgadores a ensamblar

p8-t6 1 cantidad de anillos a ensamblar

p9-t6 1 cantidad de clavos a ensamblar

p14-t6 1 cantidad de traperos en ensamble 2

t6-p15 1 cantidad de traperos almacenados en ensamble 2

p10-t7 1 cantidad de bolsas a ensamblar

p11-t7 1 cantidad de etiquetas a ensamblar

p15-t7 1 cantidad de traperos a empacar

t7-p16 1 cantidad de traperos a almacenar

p16-t8 1 cantidad de traperos a embalar 12 uds.

t8-p17 1 cantidad de traperos a almacenar 1 docena

Nota. Fuente: construcción propia. 


\section{Conclusiones}

Mediante escenarios modelados del problema real es posible discutir diferentes medidas y recursos que conlleven a la solución del mismo. Estos sirven de apoyo para tomar decisiones en la planeación de las diferentes áreas de la empresa, lo que contribuye a manejar eficientemente los tiempos de abastecimiento, producción y entrega del producto terminado, para cumplir con la producción de bienes que exige el mercado. Todo esto puede conducir al crecimiento y desarrollo del negocio.

Asimismo, la aplicación de modelos de inventarios EOQ permite integrar variables que influyen en el flujo de los abastecimientos de materiales, facilitando la obtención de datos relevantes a la realidad del negocio. Es por ello que se aconseja a la empresa utilizar e invertir en la implementación de este tipo de herramientas, para mejorar su proceso y obtener beneficios, como el saber qué cantidades de pedido debe solicitarse al proveedor y cada cuanto tiempo pedir. Esto produce la reducción de los costos de pedir materiales, almacenar inventarios, por materiales faltantes y todos aquellos que intervienen desde que el cliente genera una orden de pedido hasta que se le entrega el producto solicitado.

\section{Agradecimientos}

Principalmente a Dios por darnos la vida, a nuestros padres y familiares por el apoyo constante, al propietario de la microempresa y sus trabajadores por su colaboración e información necesaria para llevar a cabo este estudio de campo. Además, al ingeniero José Luis Ruiz Meza por su guía en el transcurso del trabajo.

\section{Referencias}

Acero, M. (27 de 09 de 2006). portafolio.com. Obtenido de http://www. portafolio.co/economia/finanzas/cadenas-suministros-globales-hoy-realidad-colombia-260936

Benchmark, B. (24 de 02 de 2016). BPR Benchmark. Obtenido de https:// nanopdf.com/download/informe-sectorial_pdf

Dinero, r. (28 de septiembre de 2017). www.dinero.com. Obtenido de http://www.dinero.com/edicion-impresa/negocios/articulo/comole-va-al-negocio-de-cosmeticos-y-aseo-en-colombia/250404 
escobaslanegra.com. (28 de 4 de 2018). escobaslanegra.com. Obtenido de http://www.escobaslanegra.com/\#!/producto/33/

Flores, F. O., Anastacio, I. S., Rodríguez, M. L., Ramírez, C. S., \& Juárez, A. G. (2011). Aanalisis de alternativas de automatización en planta de rendimientos mediante simulación. Revista de la ingenieria industrial, 5, 21.

Gaviria, J. Z., \& Zapata, G. E. (2006). Simulación de un Proceso de Producción. Revista Avances en Sistemas e Informática, Universidad Nacional de Colombia, 7.

Holguín, C. J. (2005). FUNDAMENTOS DE GESTIÓN DE INVENTARIOS. Santiago de Cali COLOMBIA: Universidad del Valle - Facultad de Ingeniería.

Jimenez, A. O., Sanchez, J. I., Martinez, G. S., \& Barriguete, J. L. (2006). La Simulación como apoyo a la optimización de procesos. informatica y calidad, 4, 5 .

Landeta, J. M., Cortés, C. B., \& Pérez, E. Z. (2015). Cálculo del punto de reorden cuando el tiempo de entrega y la demanda están correlacionados. Universidad Autónoma de San Luis Potosí Facultad de Ingeniería, México, 9.

Laveriano, W. (2010). Importancia del control de inventarios en la empresa. Lima Perú: Actualidad Empresarial.

MOHAMMAD R. AZARANG, E. G. (1996). SIMULACION Y ANALISIS DE MODELOS ESTOCASTICOS. MEXICO: MCGRAW-HILL INTERAMERICANA EDITORES. S.A. DE C.V.

RICHARD B. CHASE, F. R. (2009). ADMINISTRACIÓN DE OPERACIONES Producción y cadena de suministros. México, D.F.: McGRAW-HILL / INTERAMERICANA EDITORES, S.A. DE C.V.

Sippper, D. (1998). Planeación y control de. México, D.F.: McGRAW-HILL INTERAMERICANA EDITORES, S.A. DE C.V.

Zapata Gaviria, J., \& Peña Zapata, G. E. (2006). Simulación de un Proceso de Producción . Revista Avances en Sistemas e Informática, 7. 


\section{Apéndice A}

\begin{tabular}{cccc}
\hline \multicolumn{4}{c}{ Tabla comparación de costos EOQ y costos de } \\
$\begin{array}{c}\text { lotes arbitrarios } \\
\text { Tamaño de } \\
\text { lote (ud.) }\end{array}$ & $\begin{array}{c}\text { Costo de } \\
\text { ordenar }\end{array}$ & $\begin{array}{c}\text { Costo de } \\
\text { mantener }\end{array}$ & Costo total \\
\hline 1000 & $\$ 294,111$ & $\$ 71,401$ & $\$ 365,512$ \\
1200 & $\$ 245,092$ & $\$ 85,682$ & $\$ 330,774$ \\
1300 & $\$ 226,239$ & $\$ 92,822$ & $\$ 319,061$ \\
1400 & $\$ 210,079$ & $\$ 99,962$ & $\$ 310,041$ \\
2030 & $\$ 144,882$ & $\$ 144,945$ & $\$ 289,828$ \\
2100 & $\$ 140,053$ & $\$ 149,944$ & $\$ 289,996$ \\
\hline
\end{tabular}

Apéndice B

\begin{tabular}{cccc}
\hline \multicolumn{4}{c}{$\begin{array}{c}\text { Tabla comparación de costos EOQ y costos de lotes } \\
\text { arbitrarios }\end{array}$} \\
$\begin{array}{c}\text { Tamaño de } \\
\text { lote (ud.) }\end{array}$ & $\begin{array}{c}\text { Costo de } \\
\text { ordenar }\end{array}$ & $\begin{array}{c}\text { Costo de } \\
\text { mantener }\end{array}$ & Costo total \\
\hline 1000 & $\$ 294,111$ & $\$ 71,402$ & $\$ 365,512$ \\
1200 & $\$ 245,092$ & $\$ 85,682$ & $\$ 330,774$ \\
1300 & $\$ 226,239$ & $\$ 92,822$ & $\$ 319,061$ \\
1400 & $\$ 210,079$ & $\$ 99,962$ & $\$ 310,041$ \\
2030 & $\$ 144,882$ & $\$ 144,945$ & $\$ 289,828$ \\
2100 & $\$ 140,053$ & $\$ 149,944$ & $\$ 289,996$ \\
\hline
\end{tabular}




\section{Apéndice C}

\begin{tabular}{|c|c|c|c|}
\hline \multicolumn{4}{|c|}{$\begin{array}{c}\text { Tabla comparación de costos EOQ y costos de lotes } \\
\text { arbitrarios }\end{array}$} \\
\hline $\begin{array}{l}\text { Tamaño de } \\
\text { lote }(\mathrm{kg})\end{array}$ & $\begin{array}{l}\text { Costo de } \\
\text { ordenar }\end{array}$ & $\begin{array}{l}\text { Costo de } \\
\text { mantener }\end{array}$ & Costo total \\
\hline 5 & $\$ 176,466$ & $\$ 119,003$ & $\$ 295,469$ \\
\hline 7 & $\$ 126,047$ & $\$ 166,604$ & $\$ 292,651$ \\
\hline 10 & $\$ 88,233$ & $\$ 238,006$ & $\$ 326,239$ \\
\hline 15 & $\$ 58,822$ & $\$ 357,009$ & $\$ 415,831$ \\
\hline 20 & $\$ 44,117$ & $\$ 476,011$ & $\$ 520,128$ \\
\hline 25 & $\$ 35,293$ & $\$ 595,014$ & $\$ 630,307$ \\
\hline
\end{tabular}

\section{Apéndice D}

\begin{tabular}{cccc}
\hline \multicolumn{4}{c}{ Tabla comparación de costos EOQ y costos de lotes } \\
arbitrarios \\
$\begin{array}{c}\text { Tamaño de } \\
\text { lote (ud.) }\end{array}$ & $\begin{array}{c}\text { Costo de } \\
\text { ordenar }\end{array}$ & $\begin{array}{c}\text { Costo de } \\
\text { mantener }\end{array}$ & Costo total \\
\hline 1000 & $\$ 294,11$ & $\$ 71,40$ & $\$ 365,51$ \\
1200 & $\$ 245,09$ & $\$ 85,68$ & $\$ 330,77$ \\
1300 & $\$ 226,24$ & $\$ 92,82$ & $\$ 319,06$ \\
1400 & $\$ 210,08$ & $\$ 99,96$ & $\$ 310,04$ \\
2030 & $\$ 144,88$ & $\$ 144,95$ & $\$ 289,83$ \\
2100 & $\$ 140,05$ & $\$ 149,94$ & $\$ 290,00$ \\
\hline
\end{tabular}




\section{Apéndice E}

\begin{tabular}{cccc}
\hline \multicolumn{4}{c}{$\begin{array}{c}\text { Tabla comparación de costos EOQ y costos de lotes } \\
\text { arbitrarios de pabilo de algodón }\end{array}$} \\
$\begin{array}{c}\text { Tamaño de } \\
\text { lote }(\mathrm{kg})\end{array}$ & $\begin{array}{c}\text { Costo de } \\
\text { ordenar }\end{array}$ & $\begin{array}{c}\text { Costo de } \\
\text { mantener }\end{array}$ & Costo total \\
\hline 500 & $\$ 176,466$ & $\$ 119,002$ & $\$ 295,469$ \\
609 & $\$ 144,882$ & $\$ 144,945$ & $\$ 289,828$ \\
600 & $\$ 147,055$ & $\$ 142,803$ & $\$ 289,859$ \\
700 & $\$ 126,047$ & $\$ 166,604$ & $\$ 292,651$ \\
800 & $\$ 110,291$ & $\$ 190,405$ & $\$ 300,696$ \\
1000 & $\$ 88,233$ & $\$ 238,006$ & $\$ 326,239$ \\
\hline
\end{tabular}

Apéndice $\mathrm{F}$

\begin{tabular}{ccccc}
\hline $\begin{array}{c}\text { Materia } \\
\text { Prima }\end{array}$ & EOQ & $\begin{array}{c}\text { Resumen datos de costos EOQ } \\
\text { ordenar }\end{array}$ & $\begin{array}{c}\text { Costo de } \\
\text { mantener }\end{array}$ & Costo total \\
\hline $\begin{array}{c}\text { Pabilo de } \\
\text { algodón }\end{array}$ & 609 & $\$ 144,882$ & $\$ 144,945$ & $\$ 289,828$ \\
$\begin{array}{c}\text { Madera } \\
\text { Licra }\end{array}$ & 2030 & $\$ 144,882$ & $\$ 144,945$ & $\$ 289,828$ \\
amarre & 7 & $\$ 126,047$ & $\$ 166,604$ & $\$ 292,651$ \\
$\begin{array}{c}\text { Colgador } \\
\text { Anillos }\end{array}$ & 2030 & $\$ 144,882$ & $\$ 144,945$ & $\$ 289,828$ \\
\hline
\end{tabular}

Apéndice G

\begin{tabular}{ccccc}
\hline $\begin{array}{c}\text { Materia } \\
\text { prima }\end{array}$ & $\begin{array}{c}\text { Resumen datos de puntos de reorden EOQ } \\
\text { óptimas de } \\
\text { pedido }\end{array}$ & $\begin{array}{c}\text { Punto de } \\
\text { reorden }\end{array}$ & $\begin{array}{c}\text { Lead time } \\
\text { proveedor } \\
\text { (días) }\end{array}$ & $\begin{array}{c}\text { Tiempo } \\
\text { entre orden } \\
\text { (días) }\end{array}$ \\
\hline $\begin{array}{c}\text { Pabilo de } \\
\text { algodón }\end{array}$ & $609 \mathrm{~kg}$ & $173 \mathrm{~kg}$ & 5 & 15 \\
Madera & $2030 \mathrm{uds}$. & $705 \mathrm{uds}$. & 5 & 15 \\
Licra amarre & $7 \mathrm{~kg}$ & $3 \mathrm{~kg}$ & 5 & 15 \\
Colgador & $2030 \mathrm{uds}$. & $705 \mathrm{uds}$. & 5 & 15 \\
Anillos & $2030 \mathrm{uds}$. & $576 \mathrm{uds}$. & 5 & 15 \\
\hline
\end{tabular}

